JAHRESHEFTE DES ÖSTERREICHISCHEN ARCHÄOLOGISCHEN INSTITUTES IN WIEN

Band 78 



\title{
JAHRESHEFTE DES ÖSTERREICHISCHEN \\ ARCHÄOLOGISCHEN INSTITUTES IN WIEN
}

\author{
BAND 78
}

2009

Österreichischerlag der der Wissenschaften 
Vorgelegt von w. M. Johannes Koder in der Sitzung am 18. Juni 2010

Gedruckt mit Unterstützung des Österreichischen Archäologischen Instituts

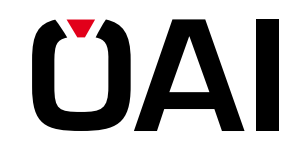

Herausgeber

Österreichisches Archäologisches Institut Franz Klein-Gasse 1

A-1190 Wien

http://www.oeai.at

Redaktionskomitee

Maria Aurenhammer

Barbara BECK-BRANDT

Johannes Koder

Michael Kerschner

Sabine LADSTÄTTER

Helga Sedlmayer

Scientifc Board

Necmi Karul, Istanbul

Stefanie Martin-Kilcher, Bern

Marion Meyer, Wien

Felix Pirson, Istanbul

Susan I. Rotroff, St. Louis, MO

R. R. R. Sмiтh, Oxford

Lutgarde VANDEPUT, Ankara

Redaktion

Barbara BECK-BRANDT

Andrea M. PÜLZ

Sigel

ÖJh

Die verwendete Papiersorte ist aus chlorfrei gebleichtem Zellstoff hergestellt, frei von säurebildenden Bestandteilen und alterungsbeständig.

Alle Rechte vorbehalten

ISSN 0078-3579

ISBN 978-3-7001-6909-3

Copyright (C) 2009 by Österreichische Akademie der Wissenschaften

Wien

Herstellung:

Satz und Layout: Andrea Sulzgruber

Druck: Prime Rate Kft., Budapest

http://hw.oeaw.ac.at/6909-3

http://verlag.oeaw.ac.at 